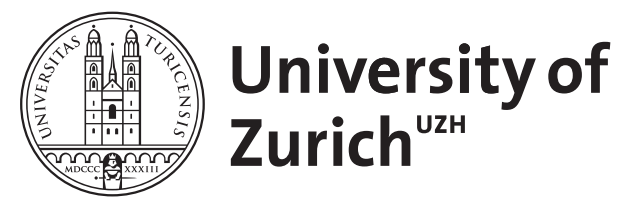

\title{
Mass transport in CuInSe2 from first principles
}

Oikkonen, L E ; Ganchenkova, M G ; Seitsonen, A P ; Nieminen, R M

\begin{abstract}
The wide scatter in experimental results has not allowed drawing solid conclusions on selfdiffusion in the chalcopyrite CuInSe2 (CIS). In this work, the defect-assisted mass transport mechanisms operating in CIS are clarified using first-principles calculations. We present how the stoichiometry of the material and temperature affect the dominant diffusion mechanisms. The most mobile species in CIS is shown to be copper, whose migration proceeds either via copper vacancies or interstitials. Both of these mass-mediating agents exist in the material abundantly and face rather low migration barriers (1.09 and $0.20 \mathrm{eV}$, respectively). Depending on chemical conditions, selenium mass transport relies either solely on selenium dumbbells, which diffuse with a barrier of $0.24 \mathrm{eV}$, or also on selenium vacancies whose diffusion is hindered by a migration barrier of $2.19 \mathrm{eV}$. Surprisingly, indium plays no role in long-range mass transport in CIS; instead, indium vacancies and interstitials participate in mechanisms that promote the formation of antisites on the cation sublattice. Our results help to understand how compositional inhomogeneities arise in CIS.
\end{abstract}

DOI: https://doi.org/10.1063/1.4799064

Posted at the Zurich Open Repository and Archive, University of Zurich ZORA URL: https://doi.org/10.5167/uzh-81916

Journal Article

Accepted Version

Originally published at:

Oikkonen, L E; Ganchenkova, M G; Seitsonen, A P; Nieminen, R M (2013). Mass transport in CuInSe2 from first principles. Journal of Applied Physics, 113(13):133510.

DOI: https://doi.org/10.1063/1.4799064 


\section{Mass transport in CulnSe $\mathrm{C}_{2}$ from first principles}

L.E. Oikkonen, ${ }^{1}$ M.G. Ganchenkova, ${ }^{1,2}$ A.P. Seitsonen, ${ }^{3}$ and R.M. Nieminen ${ }^{1}$

1) COMP Centre of Excellence, Department of Applied Physics, Aalto University, P.O. Box 11000, FI-00076 Aalto, Espoo, Finland

${ }^{2)}$ Department of Materials Science, National Research Nuclear University (MEPhI), 31 Kashirskoe Avenue, 115409 Moscow, Russia

3) Physikalisch-Chemisches Institut, University of Zürich, Winterthurerstrasse 190, CH-8057 Zürich, Switzerland

(Dated: 31 January 2013)

The wide scatter in experimental results has not allowed drawing solid conclusions on self-diffusion in the chalcopyrite $\mathrm{CuInSe}_{2}$ (CIS). In this work, the defect-assisted mass transport mechanisms operating in CIS are clarified using first-principles calculations. We present how the stoichiometry of the material and temperature affect the dominant diffusion mechanisms. The most mobile species in CIS is shown to be copper, whose migration proceeds either via copper vacancies or interstitials. Both of these mass-mediating agents exist in the material abundantly and face rather low migration barriers (1.09 and $0.20 \mathrm{eV}$, respectively). Depending on chemical conditions, selenium mass transport relies either solely on Se dumbbells, which diffuse with a barrier of $0.22 \mathrm{eV}$, or also on Se vacancies whose diffusion is hindered by a migration barrier of 2.19 eV. Surprisingly, indium plays no role in long-range mass transport in CIS; instead, indium vacancies and interstitials participate in mechanisms that promote the formation of antisites on the cation sublattice. Our results help to understand how compositional inhomogeneities arise in CIS.

PACS numbers: 61.72.Bb,66.30.-h,71.15.Mb

Keywords: Copper indium diselenide, Point defects, Diffusion, Density-functional theory

\section{INTRODUCTION}

$\mathrm{CuInSe}_{2}$ (CIS) and its quaternary analog, $\mathrm{CuIn}_{x} \mathrm{Ga}_{1 x} \mathrm{Se}_{2}$ (CIGS), have gained a foothold as absorber materials in thin-film solar cell technology. Meanwhile, the defect physics of CIS continues to puzzle researchers, and much effort has been spent on characterizing and identifying its defect structure (for a review, see e.g. ${ }^{1}$ ). Point defects in CIS play an essential role not only by influencing directly the electronic properties of the material, but also through mediating diffusion during material growth, processing, and device operation. Knowledge of these atomic-scale processes would greatly benefit device control and performance, yet no solid conclusions on the topic have been reached. Even in silicon, one of the simplest materials, the dominant diffusion mechanisms were debated for decades [ref?]. It is not surprising, then, that a material with a much more complicated structure such as CIS poses an even greater challenge for characterization.

The diffusion phenomena occurring in chalcopyrite thin-film structures have been explored experimentally by measuring concentration profiles with methods such as secondary-ion mass spectroscopy (SIMS) ${ }^{2-4}$, Auger electron spectroscopy $(\mathrm{AES})^{5}$, radioactive tracer technique $(\mathrm{RTT})^{6-8}$, and nuclear magnetic resonance $(\mathrm{NMR})^{9}$. However, extracting quantitative measures regarding self-diffusion from the experiments has proven a challenging task, and the studies can at most raise speculations of the dominant self-diffusion mechanisms in CIS: copper and indium diffusion have mostly been attributed to a vacancy-driven mechanism ${ }^{2,3,5,30}$, while selenium-related evidence is pointing towards an interstitial mechanism ${ }^{6}$.
Results should always be interpreted taking into account the limitations imposed by the methodology used. Indirect methods like SIMS and AES provide values for chemical diffusion coefficients, which by definition are affected by the presence of internal and external electric fields ${ }^{10}$. While direct methods such as RTT and NMR can be used to obtain actual self-diffusion coefficients, the data gathered so far is both too scarce and shows significant scatter to allow drawing solid conclusions on the mass transport mechanisms in CIS. The scatter in the data reflects in part differences in sample composition, conductivity $^{11}$, and experimental conditions. Different self-diffusion mechanisms may also operate in distinct temperature regimes, further complicating the interpretation of the results.

In order to fill the gap in our knowledge regarding self-diffusion in CIS, the diffusion phenomena can be approached from a computational perspective. Firstprinciples simulations offer the possibility to examine viable migration paths and mechanisms atom-wise and quantify their corresponding contributions to mass transport. A few copper-related reports on the topic have recently been published ${ }^{17,18}$, but they treat the diffusion of copper vacancies and interstitials separately and do not state conditions under which each mechanism would dominate. In the present work, we will go more in depth to the topic than previous studies, considering all three components of CIS. We use first-principles calculations to answer the following questions regarding mass transport in CIS: (1) What is the most probable mobile species? (2) How does the dominant mass transport mechanism for each component depend on the stoichiometry of the material and on the temperature? 

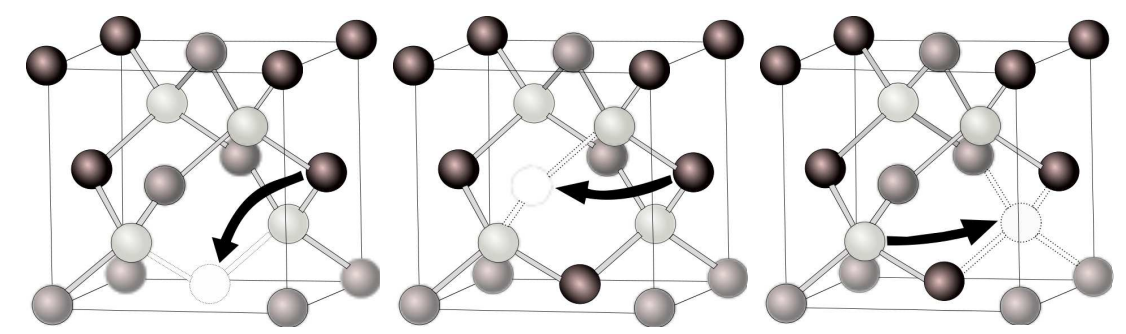

(a) $V_{\mathrm{Cu}} \rightarrow V_{\mathrm{Cu}}$

(b) $V_{\text {In }} \rightarrow \mathrm{Cu}_{\text {In }}+V_{\mathrm{Cu}}$

(c) $V_{\mathrm{Se}} \rightarrow V_{\mathrm{Se}}$

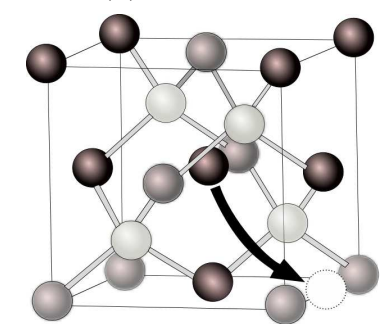

(d) $\mathrm{Cu}_{i} \rightarrow \mathrm{Cu}_{i}$

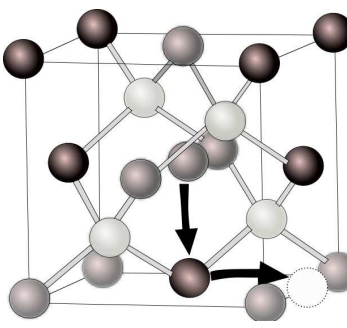

(e) $\mathrm{In}_{i} \rightarrow \mathrm{In}_{\mathrm{Cu}}+\mathrm{Cu}_{i}$

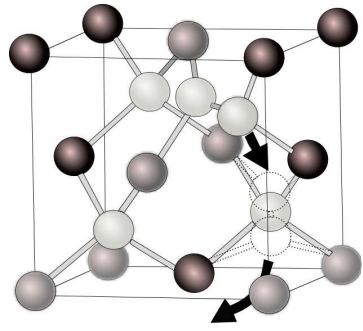

(f) $(\mathrm{Se}-\mathrm{Se})_{\mathrm{Se}} \rightarrow(\mathrm{Se}-\mathrm{Se})_{\mathrm{Se}}$

FIG. 1. Schematic diagram of the most favorable migration path for each considered defect. The copper atoms are drawn in dark color, indium atoms in grey, and selenium atoms in light color. Only one-eighth of the 64-atom supercell is shown for clarity.

\section{METHODOLOGY}

\section{A. Defining diffusion parameters}

Atomic diffusion or mass transport encompasses all diffusion by one atomic species in a compound, namely, all atoms exchanging places belong to the same species. Yet making diffusion jumps requires assistance from point defects. A defect-mediated diffusion jump typically proceeds by the exchange of an atom with a vacancy (vacancy-mediated diffusion) or by an atomic jump through interstitial space in the lattice (interstitialmediated diffusion). For instance, in order for vacancymediated diffusion to occur, the diffusing atom must meet a vacancy and overcome an activation barrier, $E_{m}$, that separates it from the vacant lattice site. After the jump, the atom waits for another vacancy in order to make the next jump. The corresponding contribution to the selfdiffusion coefficient is then the product of the vacancy concentration, $C_{V}$, and the vacancy diffusivity, $D_{V} \sim$ $\exp \left(-E_{m} / k T\right)$, describing the probability to jump. Similarly, the contribution from interstitial-mediated diffusion is $C_{I} D_{I}$, where $C_{I}$ is the concentration of interstitials and $D_{I}$ is their diffusivity. The total self-diffusion coefficient of an atomic species is the sum of all these different mechanisms $i$ : $D_{\text {self }}=\sum_{i} C_{i} D_{i}$.

Defect concentrations $C_{i}$ are sensitive to the experimental conditions. Under thermal equilibrium conditions, a defect concentration is described by the Arrhenius equation, being proportional to $\exp \left(-E_{f} / k T\right)$, where $E_{f}$ is the formation energy of the defect ${ }^{19}$. If the samples are held long enough at a certain temperature, they are expected to reach thermal equilibrium, and the defect concentrations will equilibrate accordingly - this is the case in the experimental studies that are referred to in this article. In thermal equilibrium, an activation energy of

$$
E_{a}=E_{m}+E_{f}
$$

is then required for atomic diffusion to take place. Theoretically calculated values for $E_{a}$ can be compared to experimental values derived from the slope of an Arrhenius plot of self-diffusion coefficients measured in different temperatures. It should be noted that, under nonequilibrium conditions such as during irradiation or ion implantation, the defect concentrations $C_{i}$ stay independent of temperature and Eq. 1 is reduced to the form $E_{a}=E_{m}$.

The dominant mass transport mechanism for each elemental component can vary depending on the stoichiometry of the material (through $E_{f}$ ) as well as temperature. In each compositional regime, the main mass transport mechanism is determined by the one having the lowest activation energy. The temperature dependence of the transport mechanisms is revealed through their selfdiffusion coefficients. The mechanism with the largest self-diffusion coefficient dominates in a certain temperature range.

\section{B. Computational details}

The calculations in this work have been performed using the plane-wave code $\operatorname{VASP}^{12,13}$ with the projectoraugmented wave $(\mathrm{PAW})$ method $^{14,15}$. The plane-wave basis set has been truncated with a cut-off energy of $400 \mathrm{eV}$. The exchange-correlation potential has been 
modelled with the range-separated hybrid functional HSE06 $^{16}$, which has shown considerable improvement over (semi)local functionals in the description of the atomic and electronic properties of $\mathrm{CIS}^{17,18,20}$. The portion of Hartree-Fock exchange, $\alpha$, has been set to the value of 0.25 derived from theory ${ }^{21}$.

The formation energies $E_{f}$ for each mass-mediating agent have been computed following the procedure outlined in our previous work $^{20}$, including finite-size scaling $^{22,23}$. Each defect has been considered in its relevant charge state ${ }^{20,32}$. The formation energies are sensitive to the stoichiometry of the material and, in the case of charged defects, also to the Fermi level position $\mu_{e}$.

The energetically most favorable migration route has been identified out of the different possible paths for each mass-mediating agent (details of the other possible paths will be described elsewhere ${ }^{26}$ ). The activation barriers, $E_{m}$, for the reaction paths have been computed employing the climbing-image nudged-elastic-band method $(\mathrm{CINEB})^{24}$ in a 64 -atom supercell with a $k$ point sampling of $2 \times 2 \times 2$. The structures have been allowed to relax until the forces on each atom fell below $0.01 \mathrm{eV} / \AA$. It should be noted that the value of $E_{m}$ does not depend on the stoichiometry, but is simply the difference in energy between the equilibrium and saddle-point configurations. As such, it appears to converge much faster with supercell size than mere formation energies. Additional calculations involving supercells of 144 atoms indicate that barrier values vary at most by $0.07 \mathrm{eV}$, suggesting that a 64-atom supercell is sufficient for this purpose. After the evaluation of the barriers, the self-diffusion coefficients $D\left(\mu_{e}, T\right)$ have been estimated as described $i^{25}$.

\section{RESULTS AND DISCUSSION}

\section{A. Copper mass transport}

CIS is typically grown $\mathrm{Cu}$-poor, with an even more $\mathrm{Cu}$-poor layer forming on top of the samples? ${ }^{\text {? }}$. The copper deficiency is believed to result in an abundance of copper vacancies. This expectation is supported by the remarkably low formation energy computed for $V_{\mathrm{Cu}}$ in several prior theoretical works, including ours ${ }^{17,20,27,28}$. According to our results, copper vacancies prefer to diffuse in the copper sublattice of the chalcopyrite structure with a migration barrier of $1.09 \mathrm{eV}$. The corresponding mechanism is illustrated in Figure 1(a).

Copper interstitials may co-exist with copper vacancies in the material, and they populate the octahedral sites in the lattice especially under excess $\mathrm{Cu}$ conditions. As presented in Table I, they have the lowest formation energy among the three intrinsic interstitials in CIS. A copper interstitial diffuses from one octahedral site to the next equivalent one with a migration barrier of only 0.20 $\mathrm{eV}$.

While both $V_{\mathrm{Cu}}$ and $\mathrm{Cu}_{i}$ can mediate mass transport in CIS, one of them will dominate depending on con- ditions such as stoichiometry and temperature. Comparing the activation energies of the two mechanisms at different Fermi level positions in Figure 2, it is seen that copper interstitials are the leading mediating agents for copper throughout the Fermi level range under $\mathrm{Cu}$-rich conditions. Under Cu-poor conditions, copper interstitials mediate mass transport up to Fermi level position of $0.2 \mathrm{eV}$ (i.e. $p$-type material) after which copper vacancies will prevail until pinning prevents the Fermi level from rising any further ${ }^{29}$. The impact of the two mechanisms can also be compared as a function of temperature by plotting the dependency of the corresponding self-diffusion coefficients (not shown). In the case of copper, the curves do not cross, i.e. a transition from one mechanism to another does not occur in typical growth and processing temperatures, thus leaving copper mass transport dependent only on the Fermi level position.

Experimentally determined values for the activation energies of copper have been published on several occasions, ranging from 0.2 to $1.25 \mathrm{eV}$ (see $\mathrm{se}^{17}$ and references therein). Yet as explained in the detailed analysis in ${ }^{10}$, these values mostly do not represent self-diffusion, and actual data on self-diffusion is too limited to provide a reliable value for the activation energy. The migration barrier values for copper computed in this work agree closely with the previous HSE06 calculations ${ }^{17,18}$.

Evidence supporting interstitial-driven copper transport has been accumulating little by little. From the experimental side, copper vacancies were suspected for a long time to mediate copper diffusion based on indirect evidence of presumed vacancy concentrations correlating with diffusion coefficients ${ }^{30}$. However, the experimentally determined diffusion coefficients showed such a large scatter that it started to seem like only one diffusion mechanism would not be able to account for it all. Besides vacancies, it was suggested that another mechanism such as interstitials could be operational at least under $\mathrm{Cu}$-rich conditions ${ }^{7}$. Only recently, the feasibility of $\mathrm{Cu}_{i}$ migration was demonstrated by theoretical calculations $^{18}$, which we now confirm and extend with an explicit analysis of the effect of chemical conditions and temperature.

\section{B. Selenium mass transport}

Selenium defects exist predominantly in the neutral charge state and therefore maintain a constant formation energy almost throughout the Fermi level range ${ }^{20}$. Selenium vacancies form particularly easily under Se-poor conditions with a formation energy of $1.04 \mathrm{eV}^{20}$. However, the low formation energy is counterbalanced by a high migration energy of $2.19 \mathrm{eV}$, which effectively prevents their diffusion. Such a large value probably stems from the strong lattice relaxation around $V_{\mathrm{Se}}$ : the two nearest-neighbor In atoms around the vacant site form a dimer bond $\mathrm{d}^{20,31}$ that must be broken before diffusion can occur. The migration jump of $V_{\mathrm{Se}}$ is shown schematically 
in Figure 1(c).

Extra selenium atoms are accommodated as dumbbells in the CIS lattice. A dumbbell consists of two selenium atoms sharing the same lattice site (see Ref. ${ }^{32}$ ) and can be oriented in multiple ways. Dumbbells dissociate if one of the two Se atoms jumps away. In practice, the jumping atom will end up sharing a lattice site with another Se atom, thus forming yet another dumbbell configuration as depicted in Figure 1(f). These jumps require only 0.22 $\mathrm{eV}$, indicating that extra Se atoms will be quite mobile.

Comparing the activation energies of the two mechanisms in Figure 2, selenium diffusion via dumbbells is without doubt energetically more favorable than via vacancies under Se-rich conditions. Under Se-poor conditions, however, the mechanisms have similar activation energies and therefore both can be expected to contribute to mass transport. The preferred mechanism does not depend on temperature.

Our results are in line with radioactive tracer measurements which indicate that selenium diffusion occurs via neutral selenium interstitials ${ }^{6}$. The study was conducted in single-crystal CIS when the $\mathrm{Se}_{2}$ partial pressure was varied at a high temperature. Single-crystal samples are better suited for comparison with theoretical results than polycrystalline material, which is usually dominated by grain boundary processes. Indeed, in another RTT measurement probing polycrystalline material, the prevalent self-diffusion mechanism for selenium was suspected to be grain boundary diffusion at least in the temperature range of $200-400^{\circ} \mathrm{C}^{8}$. The activation energy obtained for Se grain boundary diffusion was only $0.26 \mathrm{eV}$, while that for lattice diffusion was estimated to be $1.98 \mathrm{eV}$. The estimated value was derived from limited data from a previous study employing junction-depth measurements ${ }^{33}$ and lies somewhat lower than what has been obtained

TABLE I. Energy barriers of the most favorable migration paths $\left(E_{m}\right)$ and formation energies $\left(E_{f}\right)$ for the vacancies and interstitials in CIS. The formation energies correspond to the initial configuration before the reaction at Fermi level position equal to $0 \mathrm{eV}$. The formation energies as well as their error bars have been derived using finite-size scaling as described in our previous work ${ }^{20}$. The error bars also apply to the formation energies under $\mathrm{Cu}$-rich conditions.

\begin{tabular}{cclcc}
\multicolumn{2}{c}{ Reaction } & Charge & $E_{m}$ & $E_{f}(\mathrm{eV})$ \\
Initial & Final & state & $(\mathrm{eV})$ & $\mathrm{Cu}$-poor \\
$\mathrm{Cu}$
\end{tabular}

Vacancy-mediated

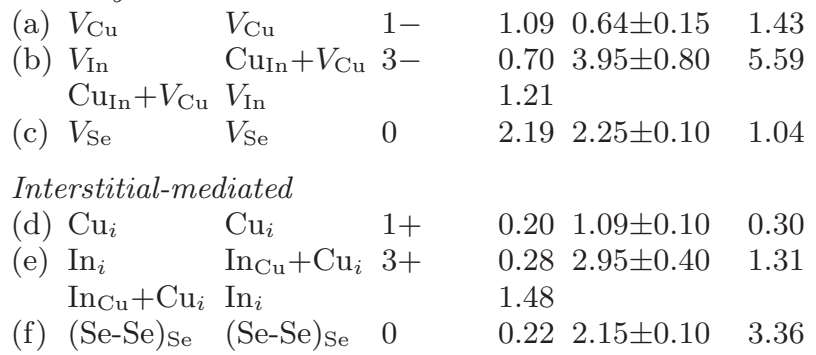

in our study. Based on these considerations, selenium mass transport probably occurs via grain boundaries in polycrystalline material and, depending on chemical conditions, either via selenium vacancies or dumbbells in single-crystal material.

\section{Indium mass transport}

Indium vacancies and interstitials are not likely to exist in CIS samples in significant quantities based on thermodynamic considerations. Their formation energies depend strongly on the Fermi level position due to their high charge states ( $3-$ for $V_{\text {In }}, 3+$ for $\left.\operatorname{In}_{i}\right)$, and are generally large apart from small, specific regions of stoichiometry and Fermi level position ${ }^{34}$.

Indium diffusion via indium vacancies or interstitials is hampered by antisite formation. For instance, $V_{\text {In }}$ would rather jump to the nearest-neighbor copper site and thus form an antisite $\mathrm{Cu}_{\text {In }}$ (migration barrier of 0.70 $\mathrm{eV})$ than to exchange places with an indium atom (1.00 $\mathrm{eV})$. The corresponding path is shown schematically in Figure 1(b). Since the final configuration of the lowestenergy jump, $\mathrm{Cu}_{\mathrm{In}}+V_{\mathrm{Cu}}$, is rather loosely bound with a binding energy of only $0.09 \mathrm{eV}, V_{\mathrm{Cu}}$ can be expected to move away from the $\mathrm{Cu}_{\text {In }}$ antisite and continue diffusing on its own. Reversing the initial reaction is somewhat less likely due to a barrier of $1.21 \mathrm{eV}$ and would still not result in mass transport.

$\mathrm{In}_{i}$ never jumps to another interstitial site, but instead heads straight to a copper site. As a result, an $\mathrm{In}_{\mathrm{Cu}}$ antisite is formed, and the copper atom is kicked out into an interstitial site (Figure 1(e)). The energy gain from the jump to the $\mathrm{Cu}$ site is so large $(1.21 \mathrm{eV})$ that all extra In atoms can be expected to eventually end up in the $\mathrm{Cu}$ sublattice. This will add to the already significant concentration of $\mathrm{In}_{\mathrm{Cu}}$ antisites that will be created due to their exceptionally low formation energy ${ }^{35}$, which we confirm. The binding energy of the $\mathrm{In}_{\mathrm{Cu}}+\mathrm{Cu}_{i}$ complex is $0.08 \mathrm{eV}$, meaning that $\mathrm{Cu}_{i}$ can quite easily detach from the antisite and start diffusing on its own. If $\mathrm{In}_{\mathrm{Cu}}$ would encounter a copper vacancy, it would be able to take isolated jumps in the copper sublattice, but the impact of this mechanism would be quite negligible on the whole. Based on these results, it can be concluded that neither indium vacancies nor interstitials will contribute to longrange mass transport in CIS.

Experimental investigations of the mobility of indium have often been carried out concurrently with gallium ${ }^{2-5}$. These two cannot be practically disentangled in a compound such as CIGS where the cation-III-atoms compete for the same sublattice positions. In and Ga diffusion has been suggested to proceed in the cation sublattice via vacancies again due to variations in diffusivity with respect to the composition of the sample ${ }^{2,3,5}$. Interestingly, the activation energy for the interdiffusion of indium in single-crystal material has only been recorded in $\mathrm{CuGaSe}_{2}\left(0.32 \mathrm{eV}^{4}\right)$ but not in CIS. This experimental 


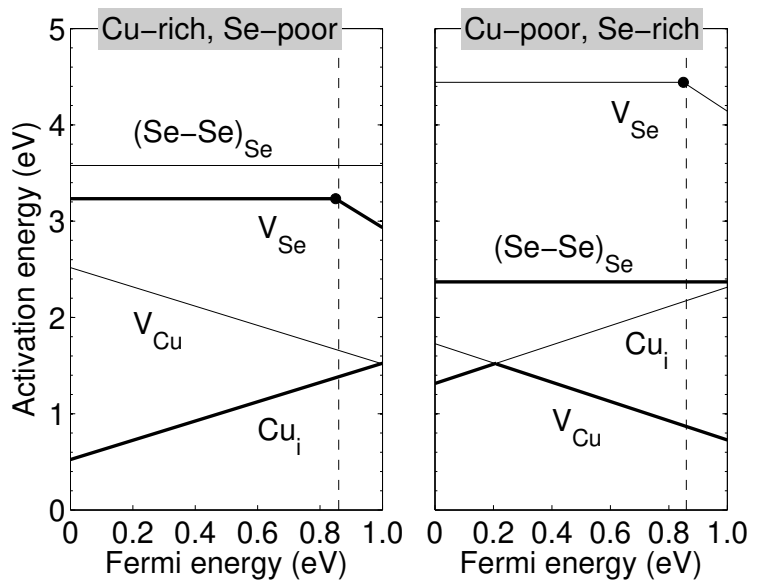

FIG. 2. Activation energies of the mass transport-mediating defects in CIS under Cu-rich/Se-poor and Cu-poor/Se-rich conditions. The dominant mass transport mechanism at each value of the Fermi level is determined by the lowest corresponding activation energy, which has been drawn in a thicker line. The dashed vertical line represents the band gap value obtained from the calculations.

evidence together with our computational results leave room for the possibility that In could behave differently in the absence of gallium, and this should be investigated further. Indium diffusion may also be governed solely by grain boundary processes in polycrystalline material, evidence for which was obtained in Ref. ${ }^{4,36}$ in the temperature range of $200-400^{\circ} \mathrm{C}$.

\section{CONCLUSIONS}

In this work, the mass transport of the three constituents in CIS has been investigated with firstprinciples calculations. Based on thermal activation energies, copper is found to be the fastest diffusing element in the material, followed by selenium. In contrast, indium will practically not move at all. These results support early experimental observations employing nuclear magnetic resonance spectroscopy (NMR) $)^{9}$ and electronbeam-induced current $(\mathrm{EBIC})^{37}$ which identified copper as the most mobile species out of the two cations in CIS at $300-350^{\circ} \mathrm{C}$. At the same time, no mobility of In was observed $^{9}$.

Copper mass transport is mediated by either copper vacancies or interstitials depending on chemical conditions. Both mediating agents exist abundantly in the material and face rather low migration barriers (1.09 and $0.20 \mathrm{eV}$, respectively). Copper interstitials mediate mass transport under $\mathrm{Cu}$-rich conditions. Under $\mathrm{Cu}$-poor conditions, mass transport occurs via interstitials up to Fermi level position of $0.2 \mathrm{eV}$, and via copper vacancies at higher electron doping levels. The leading mechanism will remain the same in typical growth and processing temperatures.

Selenium mass transport also is sensitive to the stoichiometry of the material. Selenium vacancies require only $1.04 \mathrm{eV}$ to form under Se-poor conditions, but their diffusion is hindered by a migration barrier of $2.19 \mathrm{eV}$. Extra selenium atoms are accommodated as dumbbells in the selenium sublattice and diffuse with a low barrier of $0.22 \mathrm{eV}$. Under Se-rich conditions, mass transport proceeds via dumbbells, while a competition between the two mechanisms takes place under Se-deficient conditions. Again, the preferred mechanism does not depend on temperature.

Indium mass transport is hampered by antisite formation. Due to their high formation energies, indium vacancies and interstitials would only occur in small concentrations in CIS. Additionally, it turns out that both of them prefer to occupy a copper site. Therefore, they cannot contribute to long-range mass transport.

Out of the considered defects, $V_{\mathrm{Cu}}, \mathrm{Cu}_{i}$, and Se dumbbell diffuse particularly easily in CIS due to their low migration barriers. Instead of staying isolated, they are likely to meet other defects and form clusters, or diffuse out of the samples, as well as heal radiation damages by recombination. As a result of their diffusion, compositional inhomogeneities may arise. Our results help to explain why and how CIS maintains a certain kind of microstructure. This study provides a framework for further studies of defect kinetics in CIS.

\section{ACKNOWLEDGMENTS}

This work has been supported by the Academy of Finland through its Centres of Excellence Program (20122017). The computational resources have been provided by Center of Scientific Computing (CSC), Espoo.

${ }^{1}$ S. Siebentritt, M. Igalson, C. Persson, and S. Lany, Prog. Photovolt.: Res. Appl., 18:390, 2010.

2 D.J. Schroeder, G.D. Berry, and A.A. Rockett. Appl. Phys. Lett., 69:4068-4070, 1996.

${ }^{3}$ O. Lundberg, J. Lu, A. Rockett, M. Edoff, and L. Stolt. J. Phys. Chem. Sol., 64:1499-1504, 2003.

${ }^{4}$ K. Djessas, S. Yapi, G. Massé, M. Ibannain, and J.L. Gauffier. J. Appl. Phys., 95:4111, 2004.

${ }^{5}$ M. Marudachalam, R.W. Birkmire, H. Hichri, J.M. Schultz, A. Swartzlander, and M.M. Al-Jassim. J. Appl. Phys., 82:28962905, 1997.

${ }^{6}$ H.J. von Bardeleben. J. Appl. Phys., 56:321-326, 1984.

${ }^{7}$ K. Gartsman, L. Chernyak, V. Lyahovitskaya, D. Cahen, V. Didik, V. Kozlovsky, R. Malkovich, E. Skoryatina, and V. Usacheva. J. Appl. Phys., 82:4282-4285, 1997.

${ }^{8}$ A. Praveen Kumar and K.V. Reddy. Thin Solid Films, 304:365370, 1997.

${ }^{9}$ K. Becker and S. Wagner. Phys. Rev. 27:5240, 1983.

${ }^{10}$ I. Lubomirsky, K. Gartsman, and D. Cahen. J. Appl. Phys., 83: 4678, 1998.

${ }^{11}$ M. Kleinfeld and H.-D. Wiemhöfer. Solid State Ionics, 28-30: $1111,1988$.

${ }^{12}$ G. Kresse and J. Furthmüller. Phys. Rev. B, 54:11169-11186, 1996.

${ }^{13}$ G. Kresse and J. Furthmüller. Comp. Mat. Sci., 6:15-50, 1996.

${ }^{14}$ P.E. Blöchl. Phys. Rev. B, 50:17953, 1994. 
${ }^{15}$ G. Kresse and D. Joubert. Phys. Rev. B, 59:1758, 1999.

${ }^{16}$ J. Heyd, G.E. Scuseria, and M. Ernzerhof. J. Chem. Phys., 118: 8207, 2003.

${ }^{17}$ J. Pohl and K. Albe. J. Appl. Phys., 108:023509, 2010. J. Appl. Phys., 110:109905 (E), 2010.

${ }_{18}$ J. Pohl, A. Klein and K. Albe. Phys. Rev. B, 84:121201, 2011.

19 J. Philibert. Atom Movements: Diffusion and Mass Transport in Solids, Les Ulis, France: Éditions de Physique, 1991.

${ }^{20}$ L. E. Oikkonen, M. G. Ganchenkova, A. P. Seitsonen, and R. M. Nieminen. J. Phys. Condens. Mat., 23:422202, 2011.

${ }^{21}$ J. Perdew, M. Ernzerhof, and K. Burke. J. Chem. Phys., 105: 9982, 1996.

${ }^{22}$ C.W.M. Castleton, A. Höglund, and S. Mirbt. Phys. Rev. B 73 : 035215,2006

${ }^{23}$ C.W.M. Castleton, A. Höglund, and S. Mirbt. Model. Simul. Mater. Sci. Eng. 17: 084003, (2009)

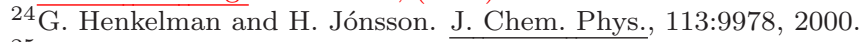

${ }^{25}$ W. Frank, U. Breier, C. Elsässer, and M. Fähnle. Phys. Rev. Lett., 77:518, 1996.

${ }^{26}$ L. E. Oikkonen, M. G. Ganchenkova, A. P. Seitsonen, and R. M. Nieminen. To be published.

${ }^{27}$ S. B. Zhang, S.-H. Wei, A. Zunger, and H. Katayama-Yoshida.
Phys. Rev. B, 57:9642, 1998.

${ }^{28}$ S. Lany and A. Zunger. J. Appl. Phys., 100:113725, 2006.

${ }^{29}$ It should be noted that the HSE06 functional employed in this work underestimates the cohesive energy of metallic copper by $0.44 \mathrm{eV}$. This may articifially lower the activation energy of $\mathrm{Cu}_{i}$ and increase the activation energy of $V_{\mathrm{Cu}}$.

${ }^{30}$ G. Dagan, T.F. Ciszek, and D. Cahen. J. Phys. Chem., 96:1100911017, 1992

${ }^{31}$ S. Lany and A. Zunger. Phys. Rev. B 72:035215, 2005.

${ }^{32}$ L. E. Oikkonen, M. G. Ganchenkova, A. P. Seitsonen, and R. M. Nieminen. Phys. Rev. B 86:165115, 2012.

${ }^{33}$ P. Migliorato, J. L. Shay, H. M. Kasper, and S. Wagner. J. Appl. Phys. 46:1777, 1975.

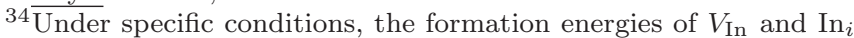
can be as low as $1.5-2 \mathrm{eV}$

${ }^{35}$ C. Persson, Y. J. Zhao, S. Lany, and A. Zunger. Phys. Rev. B 72:035211, 2005

${ }^{36}$ K. V. Reddy, A. Murty, and A. P. Kumar. Proc. Fourth Int. Symp. Proc. Phys. Mod. Semicond. Techn. 96:237-238, 1996.

37 S. Soltz, G. Dagan, and D. Cahen. Solid State Ionics 28-30:1105, 1988. 